\title{
Serial Cognition and Personality in Macaques
}

\author{
Drew M. Altschul ${ }^{1,2,{ }^{*}}$, Herbert S. Terrace ${ }^{3}$, \& Alexander Weiss ${ }^{1,2}$
}

\author{
${ }^{1}$ The University of Edinburgh \\ ${ }^{2}$ Scottish Primate Research Group \\ ${ }^{3}$ Columbia University \\ *Corresponding author (Email: dmaltschul@gmail.com)
}

Citation - Altschul, D. M., Terrace, H. S., \& Weiss, A., (2016). Serial cognition and personality in macaques. Animal Behavior and Cognition, 3(1), 46-64. doi: 10.12966/abc.02.04.2016

\begin{abstract}
We examined the associations between serial cognition and personality in rhesus macaques (Macaca mulatta). Nine macaques were tested on a simultaneous chaining task to assess their cognitive abilities. They were also rated for personality traits and scored according to a previously extracted six component structure derived from free-ranging rhesus macaques. Friendliness and Openness were positively associated with good performance on three measures of accuracy on the serial learning task: Progress, Error, and Rewarded (i.e., correctly completed) Trials. Faster Reaction Times were associated with lower Friendliness and higher Confidence, as well as higher Openness when only correct responses were analyzed. We also used regularized exploratory factor analysis to extract two, three, four, five, and six factor structures, and found consistent associations between accuracy and single factors within each of these structures. Prior results on intelligence in other nonhuman primate species have focused on basic intelligence tests; this study demonstrates that more complex, abstract cognitive tasks can be used to assess intelligence and personality in nonhuman primates.
\end{abstract}

Keywords - Primates, Rhesus macaques, Personality, Individual differences, Serial cognition, Comparative cognition

The study of individual psychological differences in animals originated with Pavlov, who classified personality as learning characteristics among his dogs (Pavlov, 1908/1941). In primates, Yerkes (1939), Crawford (1938), and Hebb (1946) all worked with the same group of chimpanzees, and all three found evidence for personality, from both observer ratings and behavioral codings. Early researchers did not restrict their studies to captive animals; free-ranging Japanese macaques (Itani, 1957) and chimpanzees (Goodall, 1990) were also described as having distinct personalities.

Personality describes individual differences in behavior that are stable over time and across different contexts (Réale, Reader, Sol, McDougall, \& Dingemanse, 2007). Individual differences in cognitive abilities differ in that they rely on quantifying performance (Griffin, Guillette, \& Healy, 2015), e.g., percentage correct out of a set number of trials, or average reaction time. Serial cognition is one faculty that has been studied as a means of understanding how animals learn and manipulate complex information (D'Amato \& Colombo, 1990). However, few studies of serial cognition, as well as animal cognition more broadly (Griffin et al., 2015), have addressed where individual differences in performance come from, as the focus has typically been on the abilities of the species and not those of individuals (Herrmann, Call, Hernández-Lloreda, Hare, \& Tomasello, 2007; Terrace, 1993). The exceptions include Vonk and Povinelli (2011), who found that different chimpanzees excelled in social and physical tasks, except for one individual who performed well at both, and Herrmann, Hernández-Lloreda, Call, Hare, and 
Tomasello (2010), who reanalyzed their earlier data to assess individual differences with factor analytic techniques. The majority of these studies have focused on a single species: the common chimpanzee.

The study of animal personality, on the other hand, has flourished in the last two decades. One way in which species personalities can be described is by quantifying traits along a small number of dimensions. In humans this gave rise to the "Big Five" or "Five-Factor Model," which incorporates dimensions usually named Neuroticism, Extraversion, Openness to experience, Agreeableness, and Conscientiousness (McCrae \& John, 1992). Chimpanzees, which share a recent common ancestor with humans, possess six dimensions, five resembling the human personality dimensions, plus the dimension Dominance (King \& Figueredo, 1997). On the other hand, rhesus macaques, representatives of an older ancestor, also have six personality dimensions, that differ some from the chimpanzee and human dimensions: Anxiety, Activity, Openness, Friendliness, Confidence, and Dominance (Weiss, Adams, Widdig, \& Gerald, 2011).

Within-species personality variation may drive the variability within cognitive capacities. Female rhesus macaques with a tendency towards exploratory behaviors acquired operant responses $50 \%$ more often than less adventurous subjects (Coleman, Tully, \& McMillan, 2005); exploratory behaviors in mice covaried with learning differences (Matzel et al., 2003), and chickadees that were slow to explore were more accurate during testing, but did not learn the experimental task more quickly than other chickadees (Guillette, Hahn, Hoeschele, Przyslupski, \& Sturdy, 2015). The common attribute of these studies 'exploration' - calls to mind two human personality dimensions: Extraversion and Openness. However, most research has associated animal personality with behavior (e.g., Capitanio, 1999; Konečná et al., 2008; Pederson, King, \& Landau, 2005), rather than cognitive ability. Moreover, prior research has been largely observational (Konečná et al., 2008; Pederson et al., 2005), not experimental. Meanwhile, complex, repeatable tasks have been realized in captive environments thanks to modern computing power and equipment (Fagot, Gullstrand, Kemp, Defilles, \& Mekaouche, 2014). These tasks provide rich data that permit stronger inference about cognitive function than earlier operant techniques.

Recent studies have begun bridging the gaps between cognitive and personality psychology (Herrelko, Vick, \& Buchanan-Smith, 2012; Morton, Lee, \& Buchanan-Smith, 2013) with factor analytic approaches common to personality and intelligence research (Herrmann et al., 2010; Hopkins, Russell, \& Schaeffer, 2014). Intelligence is a general cognitive ability that underlies individual differences in performance on mental tests, such as Raven's Standard Progressive Matrices and the National Adult Reading Test (Deary, 2001). This general factor of intelligence or ' $g$ ' factor is widely used in individual differences research, and moderately sized relationships have been found between measures of $g$ and the Five-Factor Model in humans (Austin, Deary, \& Gibson, 1997). In particular, high Openness to experience has been repeatedly linked to high $g$ factor scores (DeYoung, 2014).

The majority of studies on general primate intelligence have been meta-analyses (Deaner, Van Schaik, \& Johnson, 2006; Reader, Hager, \& Laland, 2011; Schmitt, Pankau, \& Fischer, 2012) that identify intelligence differences between species. Herrmann et al. (2010) compared chimpanzees with human children; principal components analyses indicated that a 'Spatial' and a 'Physical-Social' factor best explained the structure of differences in chimpanzee performance. Subsequently, Hopkins et al. (2014) used the Primate Cognition Test Battery (Herrmann et al., 2010) modeled performance on the task as arising from a single $g$ factor, with four constructs beneath. The chimpanzee $g$ factor was heritable (Hopkins et al., 2014), as has been shown in humans (Davies et al., 2011; Deary, Spinath, \& Bates, 2006).

Serial cognition has been studied in many species with many paradigms; varying demonstrations of proficiency have been displayed across studies (McGonigle \& Chalmers, 2006). The Simultaneous Chaining (SimChain) paradigm is one of the most difficult tests of serial cognition: the commonly used serial cognition paradigm, Transitive Inference (TI), only requires binary responses, whereas SimChain requires multiple successive, correct responses for an animal to be rewarded. Monkeys' behavior in completing SimChain trials is known for defying traditional chaining theory (Ebbinghaus, 1913/2014). Instead of learning associations between successive items, monkeys learn the ordinal positions of individual items, encoding them in a spatial representation (Chen, Swartz, \& Terrace, 1997). 
Serial intelligence is a broadly applied, flexible ability: despite their differences in difficulty, the SimChain and TI paradigms share a common mental representation (Jensen, Altschul, Danly, \& Terrace, 2013). Transitive reasoning is in turn linked to symbolic manipulation (D’Amato \& Colombo, 1990), social dominance and navigation in primate hierarchies (Paxton et al., 2010), and language (Jensen et al., 2013). These links make SimChain a strong candidate for testing general cognitive ability in animals.

While the evolution of serial cognition is well documented (McGonigle \& Chalmers, 2006), why individual personalities have been selected for remains an open question (Bouchard \& Loehlin, 2001). Moreover, the evolutionary genetics underlying individual differences in intelligence and personality need not be very similar. If the contributions of gene and environment differ between personality and intelligence (Penke, Denissen, \& Miller, 2007), then how should we expect animals' personalities to vary with cognitive abilities? In nine rhesus macaques, we collected cognitive and personality data, and in a series of exploratory analyses we examined connections between personality and serial cognition, with the expectation that Openness, and possibly other macaque personality dimensions, would be associated with performance on the SimChain task.

\section{Method}

\section{Subjects}

Nine male captive-born rhesus macaques, aged 12 to 16 years, and housed at the New York State Psychiatric Institute, performed a SimChain task and were evaluated for personality. The colony was maintained in accordance with guidelines issued by the National Institutes of Health and the Institutional Animal Care and Use Committees at the New York State Psychiatric Institute and Columbia University. Macaques were individually housed in adjoining cages at the time of the study, but had been pair housed previously. Macaques were given water ad libitum, and fed commercial primate biscuits and varied fresh fruits and vegetables daily, in addition to any pellets they received as rewards in experimental tasks.

\section{Apparatus}

The apparatus was identical to that used in prior studies (Jensen et al., 2013). Testing took place in chambers housed in sound-attenuated booths. Chambers were equipped with speakers, and a pellet dispenser (Med Associates; pellets by BioServ, $190 \mathrm{mg}$ ). A computer with a touch-sensitive monitor presented stimuli and detected responses.

\section{Procedure}

The SimChain paradigm presents an ordered list as a simultaneously displayed set of images on a touchscreen monitor. A trial is completed by selecting each stimulus in the correct order (see Figure 1; Terrace, 1993). In this experiment, subjects had to learn a novel four-item list composed of arbitrary color images, each day. Subjects were given 40 trials to learn each list, which could only be accomplished through trial and error. On successful trials, subjects were rewarded with a banana pellet. On unsuccessful trials they received a $4 \mathrm{~s}$ timeout. We gathered 20 days of data, that is, 20 sessions of 40 trials each.

Because subjects had been extensively trained on SimChain tasks, no task learning effects were expected to confound results. Subjects could be expected to display their asymptotic level of performance.

\section{Personality Ratings}

Subjects were independently rated by 10 animal care volunteers using the Hominoid Personality Questionnaire (Weiss et al., 2009). The questionnaire consisted of 54 adjectives followed by 1 to 3 sentences defining adjectives in terms of everyday nonhuman primate behaviors. Items were rated on a 7point scale. Raters were familiar with subjects prior to evaluating them, but unaware of the details of 
individual subjects' performance. Raters had between 6 months and 3 years of experience with the animals; each rater typically spent several hours, one day a week, looking after the animals within the colony setting.

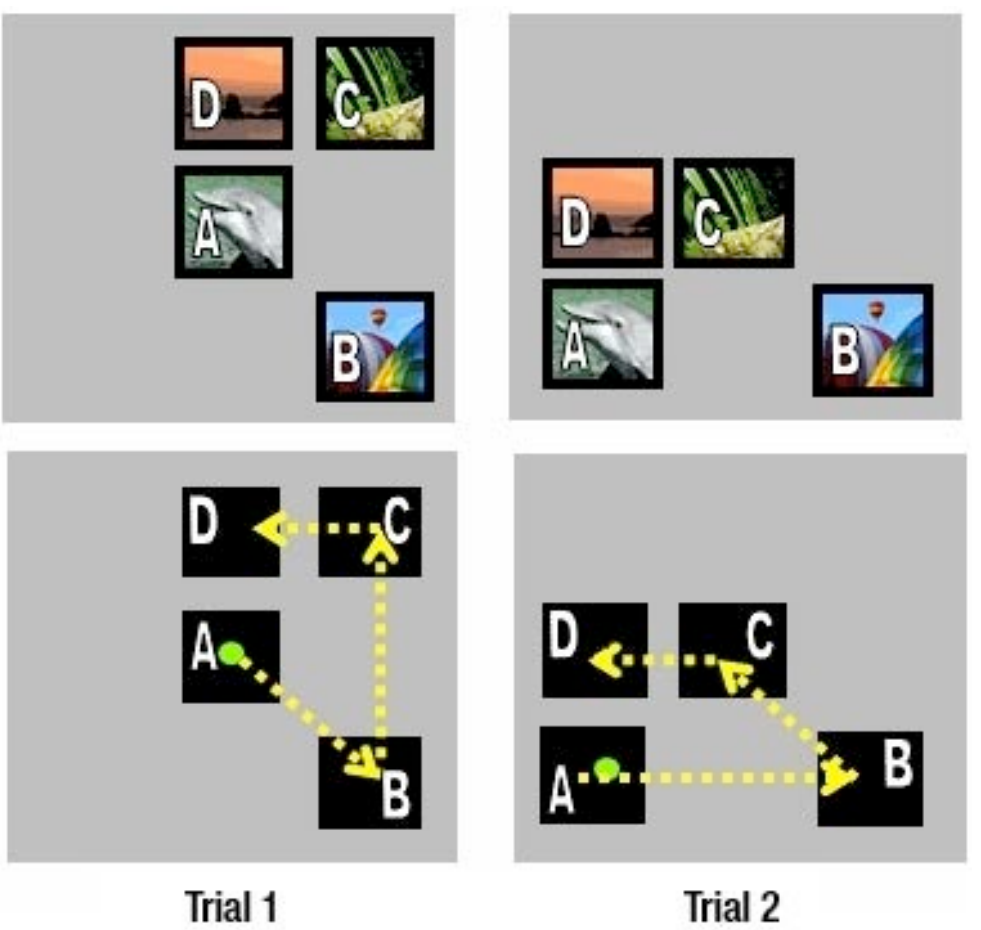

Figure 1. The Simultaneous Chaining paradigm. The task was to touch the items in the prescribed order, regardless of their positions on the screen. An example of a 4-item list is shown in two different, random arrangements, as might appear during any trial in a session. The top row shows the arrangement of ordered pictures, and the bottom row indicates the correct path of selection.

\section{Analysis}

The R programming language (version 3.2.1; R Core Team, 2015) was used for all correlation and regression analyses, using the 'psych' (Revelle, 2015), 'nlme' (Pinheiro, Bates, DebRoy, Sarkar, \& R Core Team (2015), 'Ime4' (Bates, Maechler, Bolker, \& Walker, 2015), and 'car' (Fox \& Weisberg, 2011) packages. Regularized exploratory factor analyses (Jung \& Lee, 2011) were carried out in MATLAB 2014a, using custom code by Sunho Jung.

\section{Results}

\section{Interrater Reliability}

Interrater reliabilities of personality items were calculated from all animals and all raters using intraclass correlations (Shrout \& Fleiss, 1979) $I C C(3,1)$ and $\operatorname{ICC}(3, k)$. The items 'cautious,' 'defiant,' 'independent,' and 'stingy/greedy' had ICCs less than zero and were removed from further analysis. The items 'autistic' and 'unperceptive' were omitted because both were removed from an earlier study for being unreliable and thus not included in the definitions of the components (Weiss et al., 2011). The remaining items' $I C C$ s ranged from 0.009 to 0.290 for $\operatorname{ICC}(3,1)$, and 0.079 to 0.801 for $\operatorname{ICC}(3, k)$. 


\section{Personality and Performance}

Average questionnaire ratings were used to compute domain scores from the unit-weighted matrix based on previously derived component loadings (Weiss et al., 2011; Table 1). Performance was measured using three measures of trial-by-trial accuracy. Rewarded trials reflect the binary successes and failures across each subject's trial-by-trial performances: to be rewarded, a subject must complete a full SimChain trial without error. Progress quantifies how far into the list the subject made it on any given trial, before either making an error or completing the trial. Error is defined as the amount of deviation, from the next correct response, in a subject's terminal choice. Error can be either positive or negative: If the subject makes forwards error, jumping ahead in the chain, the Error is positive. If the subject makes a backwards Error, it is negative. If the subject presses each item in the correct order and completes a trial successfully, the Error is 0. Error and Progress for each of the nine monkeys is shown in Figures 2 and 3, respectively.

Reaction time (RT) is the natural logarithm of the interval between the onset of the visual stimuli and the first response. SimChain completion utilizes a series of planned responses (Scarf, Danly, Morgan, Colombo, \& Terrace, 2011), but apart from the pause before the initial response, wherein the chain planning pauses occur depends on the individual animal. We analyzed the first response RT for only correct responses, as well as the RT for all first responses, to search for speed-accuracy trade-offs (Prinzmetal, McCool, \& Park, 2005).

Correlations between personality and performance (averaged across trials and sessions) are shown in Table 1. Friendliness was significantly positively correlated with Rewarded trials and Progress; negatively correlated with Error. Openness was significantly correlated with Progress and Error, in the same directions as with Friendliness. No significant correlations were found between personality domains and either RT measure.

Table 1

Correlations among Personality and Averaged Performance Variable

\begin{tabular}{|c|c|c|c|c|c|c|c|c|c|c|}
\hline & Anx & Act & Frd & Dom & Opn & Con & Rwd & Err & Prg & RT \\
\hline Act & 0.57 & & & & & & & & & \\
\hline Frd & -0.12 & 0.49 & & & & & & & & \\
\hline Dom & 0.70 & 0.85 & 0.08 & & & & & & & \\
\hline Opn & 0.79 & 0.63 & 0.33 & 0.50 & & & & & & \\
\hline Con & -0.53 & 0.31 & 0.57 & 0.15 & 0.26 & & & & & \\
\hline Rwd & 0.21 & 0.55 & 0.71 & 0.26 & 0.65 & 0.38 & & & & \\
\hline Err & -0.25 & 0.53 & -0.70 & -0.28 & -0.67 & -0.34 & -0.99 & & & \\
\hline Prg & 0.24 & 0.56 & 0.73 & 0.27 & 0.67 & 0.35 & 1.00 & -0.99 & & \\
\hline RT & 0.07 & 0.24 & 0.28 & 0.14 & -0.07 & -0.09 & -0.09 & 0.03 & 0.05 & \\
\hline RT1 & -0.12 & 0.19 & 0.17 & 0.09 & -0.25 & 0.08 & -0.07 & 0.02 & 0.03 & 0.91 \\
\hline
\end{tabular}

\section{Regression Analyses}

Simple correlations between averages fail to capture the nuance in individuals' performance. For example, both Error (Figure 2) and Progress (Figure 3) demonstrate learning curves and asymptotic plateaus in performance, which differ between animals. To explore personality's relationship with 
performance in more detail, we modeled each performance metric including personality predictors based on the strength of associations seen in the correlation matrix.
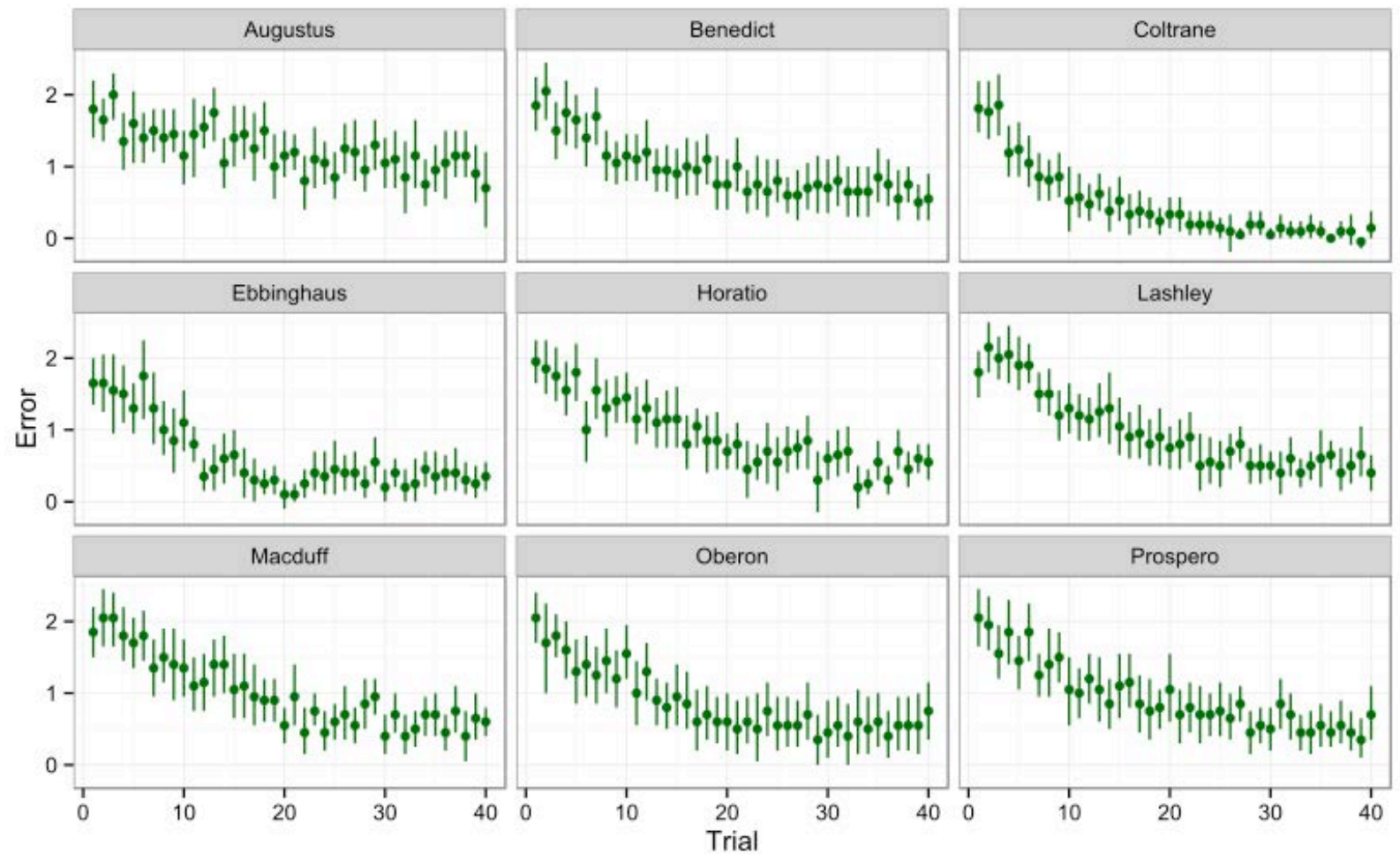

Figure 2. Average Error data from 20 sessions of 40 trial SimChain, from 9 monkeys. Bars indicate standard errors.

\section{Error}

If one wishes to model Error with linear regression, the Error data must first be transformed, because they are non-linear (Figure 2). This poses a challenge because Error can be both positive and negative, thus log-transformation is not appropriate. Fortunately, Yeo-Johnson transformation, which was designed for and tested on cases such as ours, handles negative values (Yeo \& Johnson, 2000). We constructed a series of linear mixed models, using a forward selection approach, starting with a null model which included a trial number variable and intercept. Results of our model selection are shown in Table 2.

Log-likelihood indicated that model 7 was the best fit to the data, while the small-sample corrected Akaike Information Criterion (AICc) indicated that model 6 was the best fit. The Bayesian Information Criterion (BIC) indicated that the null model was the best fit, which was a consistent prediction across all our models. The BIC is more strongly biased towards models with fewer degrees of freedom, for as the sample size increases, the probability that BIC selects the correct model approaches 1 (Vrieze, 2012). For smaller sample sizes, BIC necessarily performs less well on average, so while we continued to calculate it for all models for diagnostic purposes, we did not factor it into our selection procedures.

The details of models 6 and 7 are shown in Table 3. Both models consistently show that higher Openness was significantly associated with a smaller starting error, itself an indicator of better performance. The interaction between Friendliness and Trial was also significant in both models, similarly suggesting that Friendliness was associated with smaller error as sessions progressed. Outside of the interaction, Friendliness was not a significant predictor, though it did appear to marginally improve 
the fit of the model. The effect size of the Openness coefficient was also larger than either Friendliness coefficient.
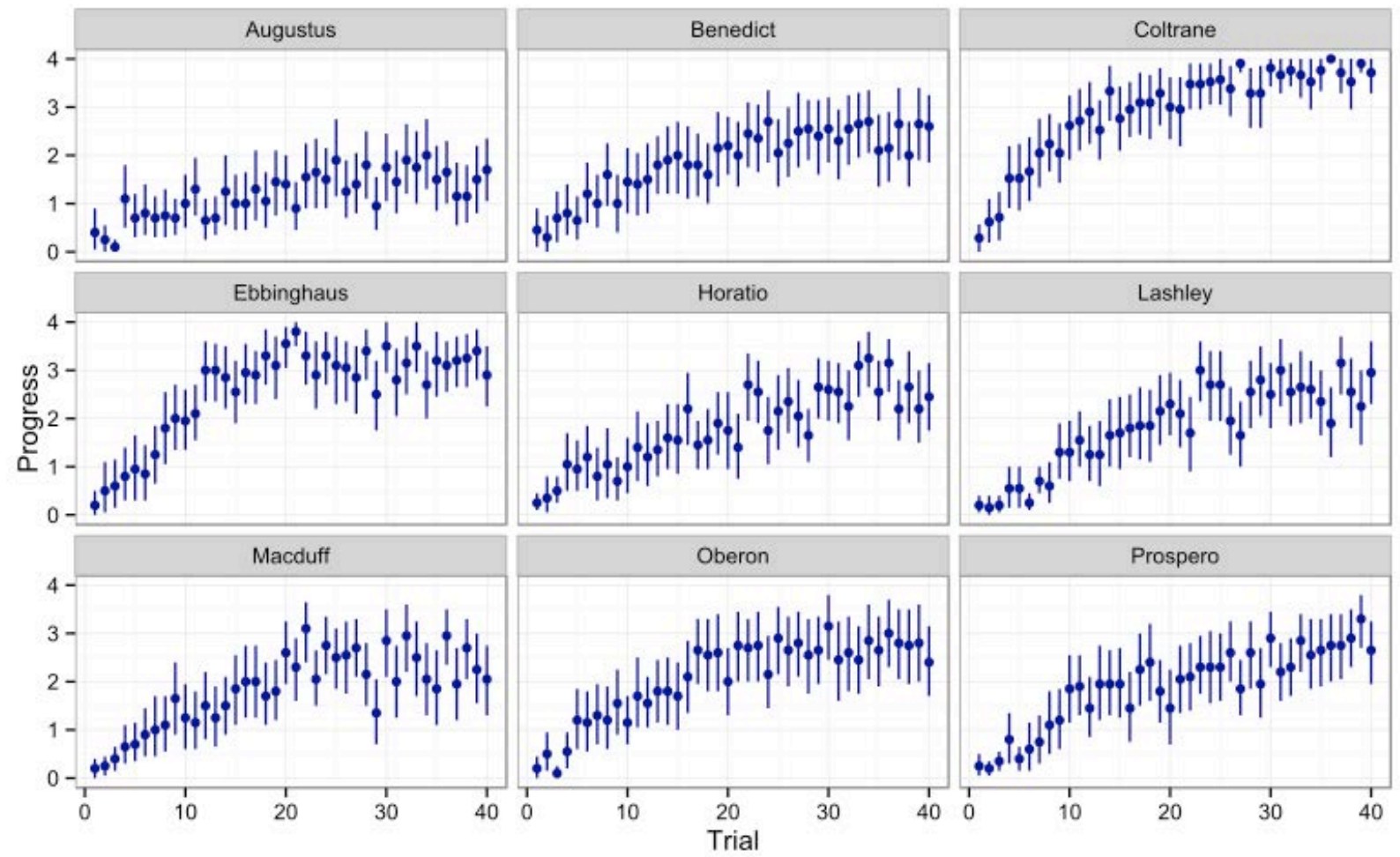

Figure 3. Average Progress data from 20 sessions of 40 trial SimChain, in 9 monkeys. Bars indicate standard errors.

Table 2

\section{Model Selection Results for Regressions Predicting Transformed Error Variable}

\begin{tabular}{|c|c|c|c|c|c|c|c|}
\hline Model Number & Variables & $\mathrm{df}$ & $\mathrm{AICc}$ & $\mathrm{BIC}$ & LogLik & $\Delta \operatorname{LogLik}$ & RM \\
\hline 0 & (Trial, Incercept) & 9 & 18117.0 & 18179.0 & -9049.5 & & \\
\hline 1 & Frd & 10 & 18117.0 & 18185.8 & -9048.5 & 1.0 & 0 \\
\hline 2 & Opn & 10 & 18115.1 & 18183.9 & -9047.5 & 1.0 & 1 \\
\hline 3 & Frd * Trial & 10 & 18112.5 & 18181.4 & -9046.2 & 1.3 & 2 \\
\hline 4 & Opn $*$ Trial & 10 & 18117.9 & 18186.7 & -9048.9 & -2.7 & 3 \\
\hline 5 & Frd $*$ Trial, Frd & 11 & 18113.5 & 18189.2 & -9045.7 & 0.5 & 3 \\
\hline 6 & Frd $*$ Trial, Opn & 11 & 18110.1 & 18185.8 & -9044.0 & 1.7 & 5 \\
\hline 7 & Frd $*$ Trial, Opn, Frd & 12 & 18111.8 & 18194.4 & -9043.9 & 0.1 & 6 \\
\hline
\end{tabular}

Note. Bolding indicates the best model, according to the procedure. $\mathrm{df}=$ degrees of freedom, AICc $=$ Akaike Information Criterion corrected for small samples, BIC $=$ Bayesian Information Criterion, LogLik $=$ Log-likelihood, $\Delta$ LogLik $=$ difference in log-likelihood between current model and last best fitting model, $\mathrm{RM}=$ the reference model for the $\Delta$ LogLik comparison. 
Table 3

Details of Linear Regression Models of Interest, Predicting Error from Personality

\begin{tabular}{lcccccc}
\hline \multicolumn{3}{c}{ Model 6} & \multicolumn{2}{c}{ Model 7} \\
\hline Predictor & $b$ & $95 \% C I$ & $\chi^{2}$ & $b$ & $95 \% C I$ & $\chi^{2}$ \\
\hline Intercept) & 3.01 & {$[1.72,4.30]$} & & 3.24 & {$[1.72,4.75]$} & \\
Trial & 0.02 & {$[-0.01,0.05]$} & $556.80 * * * *$ & 0.01 & {$[-0.02,0.05]$} & $600.00 * * * *$ \\
Opn & -0.42 & {$[-0.76,-0.08]$} & $5.71 *$ & -0.39 & {$[-0.74,-0.03]$} & $4.59 *$ \\
Frd & & & & -0.09 & {$[-0.39,0.23]$} & 3.34 \\
Frd $*$ Trial & -0.01 & {$[-0.02,-0.00]$} & $10.80 * * * *$ & -0.01 & {$[-0.02,-0.00]$} & $7.72 * *$ \\
\hline
\end{tabular}

Note. $p$-values are from Wald's $\chi^{2}$ tests. $\mathrm{CI}=$ confidence interval, Opn $=$ Openness, Frd $=$ Friendliness.

$* p<0.05 . * * p<0.01 . * * * * p<0.001$.

\section{Progress}

Progress displays a similar curve as Error ( $c f$. Figures 2 and 3), but unlike Error, it does not take negative values. However, Progress on the SimChain task can be modeled using Thurstone's learning curve (Jensen et al., 2013), so rather than linearize the Progress data, we modeled Progress with a nonlinear logistic regression. A simple logistic growth curve has three parameters, and is defined:

$$
f(x)=\frac{L}{1+e^{-k\left(x-x_{0}\right)}}
$$

where $L$ is the maximum value or asymptote of the curve, $k$ is the steepness of the curve, and $x_{0}$ is the $x$ value midpoint of the curve, also known as a scaling parameter.

We used a forward selection approach to model building, jointly inputting personality dimensions as predictors of two logistic parameters: asymptote $-L$, and steepness $-k$ (Table 4 ). The model including Friendliness alone was the best fit, but only in the most marginal sense, as the AICc and log-likelihood values were extremely close to those generated by model 2, wherein Openness was the lone personality predictor. Fit became considerably worse when both Friendliness and Openness were included, but we still wished to examine if and how their contribution to the model might change in each other's presence.

All three non-null models are described in Table 5. Friendliness was positively and significantly associated with the asymptotic level of performance; Openness negatively and significantly associated with the steepness coefficient. Due to software limitations, steepness needed to be modeled as $1 / k$, thus higher Openness was associated with a steeper, and faster, rate of learning.

\section{Rewarded Trials}

Monkeys were reinforced with food only after correctly completing a full SimChain. To model personality's impact on this binary variable, we fitted a generalized linear mixed model, with a binomial logistic link function. Model building was again carried out with a forward selection procedure, and because of the simplicity in adding individual predictors, we chose to input a broader choice of personality predictors (Table 6).

Models 5 and 7 appeared to be the best fit, according to AICc and log-likelihood, respectively. Comparing those two models (Table 7) revealed that when only Friendliness and Openness were included, both were positively associated with subjects' rate of reward. However, when all personality 
predictors were included, only Confidence showed a significant (and positive) relationship with rate of reward.

Table 4

Model Selection Results for Regressions Predicting Progress Variable

\begin{tabular}{llllllll}
\hline Model Number & Variables & df & AICc & BIC & LogLik & $\Delta$ LogLik & RM \\
\hline 0 & (Intercepts only) & 10 & 26224.8 & $\mathbf{2 6 2 9 3 . 7}$ & -13102.4 & & \\
1 & Frd & 12 & $\mathbf{2 6 2 2 2 . 6}$ & 26305.2 & -13099.3 & $\mathbf{3 . 1}$ & 0 \\
2 & Opn & 12 & 26222.8 & 26305.4 & -13099.4 & -0.1 & 1 \\
3 & Frd, Opn & 14 & 26237.6 & 26333.9 & -13104.8 & -5.5 & 1 \\
\hline
\end{tabular}

Note. Bolding indicates the best model, according to the procedure. See Table 2 for explanation of abbreviations.

Table 5

Details of Non-Linear Regression Models of Interest, Predicting Progress

\begin{tabular}{|c|c|c|c|c|c|c|c|c|c|}
\hline \multirow[b]{2}{*}{ Predictor } & \multicolumn{3}{|c|}{ Model 1} & \multicolumn{3}{|c|}{ Model 2} & \multicolumn{3}{|c|}{ Model 3} \\
\hline & $b$ & $95 \% C I$ & $t$ & $b$ & $95 \% C I$ & $t$ & $b$ & $95 \% C I$ & $t$ \\
\hline Asymptote & & & & & & & & & \\
\hline (Intercept) & -0.30 & {$[-2.27,1.66]$} & -0.30 & 2.34 & {$[-0.21,4.88]$} & 1.80 & -5.70 & {$[-8.99,-2.35]$} & $-3.35 * * * *$ \\
\hline Friendliness & 0.74 & {$[0.25,1.23]$} & $2.98 * * *$ & & & & 1.16 & {$[0.50,1.82]$} & $3.45 * * * *$ \\
\hline Openness & & & & 0.08 & {$[-0.58,0.75]$} & 0.24 & 0.98 & {$[0.16,1.79]$} & $2.35 *$ \\
\hline Steepness & & & & & & & & & \\
\hline (Intercept) & -1.47 & {$[-9.71,6.76]$} & -0.35 & 18.20 & {$[11.50,24.8]$} & $5.34 * * * *$ & 14.50 & {$[3.42,25.5]$} & $2.57 *$ \\
\hline Friendliness & 1.72 & {$[-0.31,3.75]$} & 1.66 & & & & 1.00 & {$[-0.92,2.92]$} & 1.02 \\
\hline Openness & & & & -3.41 & {$[-5.08,-1.73]$} & $-3.99 * * * *$ & -3.53 & {$[-5.29,-1.77]$} & $-3.93 * * * *$ \\
\hline Midpoint & 9.68 & {$[8.66,10.7]$} & $18.60 * * * *$ & 9.63 & {$[8.54,10.7]$} & $17.30 * * * *$ & 8.80 & {$[8.27,9.33]$} & $32.60 * * * *$ \\
\hline
\end{tabular}

Note. $p$-values are from Welch's $t$ tests.

$* p<0.05 . * * p<0.01 . * * * p<0.005 . * * * * p<0.001$.

Table 6

Model Selection Results for Regressions Predicting Rewarded Trials

\begin{tabular}{|c|c|c|c|c|c|c|c|}
\hline Model Number & Variables & $\mathrm{df}$ & $\mathrm{AICc}$ & $\mathrm{BIC}$ & LogLik & $\Delta$ LogLik & RM \\
\hline 0 & (Trial, Intercept) & 4 & 7462.1 & 7489.6 & -3727.0 & & \\
\hline 1 & Opn & 5 & 7458.7 & 7493.1 & -3724.3 & 2.7 & 0 \\
\hline 2 & Frd & 5 & 7456.7 & 7491.2 & -3723.4 & 0.9 & 1 \\
\hline 3 & Con & 5 & 7462.6 & 7491.1 & -3726.3 & -2.9 & 2 \\
\hline 4 & Act & 5 & 7460.1 & 7494.6 & -3725.1 & -1.7 & 2 \\
\hline 5 & Frd, Opn & 6 & 7453.2 & 7494.5 & -3720.6 & 2.8 & 2 \\
\hline 6 & Frd, Opn, Act & 7 & 7455.2 & 7503.4 & -3720.6 & 0.0 & 5 \\
\hline 7 & Frd, Opn, Act, Con, Dom, Anx & 10 & 7455.3 & 7524.1 & -3717.6 & 3.0 & 5 \\
\hline
\end{tabular}


Table 7

Details of Binomial Regression Models of Interest, Predicting Rewarded Trials from Personality

\begin{tabular}{|c|c|c|c|c|c|c|}
\hline \multicolumn{4}{|c|}{ Model 5} & \multicolumn{3}{|c|}{ Model 7} \\
\hline Predictor & $b$ & $95 \% C I$ & $z$ & $b$ & $95 \% C I$ & $z$ \\
\hline (Intercept) & -14.30 & {$[-18.80,-9.86]$} & & -22.80 & {$[-30.50,-15.10]$} & \\
\hline Trial & 0.09 & {$[0.08,0.09]$} & $30.70 * * * *$ & 0.09 & {$[0.08,0.09]$} & $30.70 * * * *$ \\
\hline Anxiety & & & & 1.76 & {$[-0.91,4.43]$} & 1.29 \\
\hline Activity & & & & -0.20 & {$[-2.00,1.60]$} & -0.22 \\
\hline Confidence & & & & 2.45 & {$[0.47,4.44]$} & $2.42 *$ \\
\hline Dominance & & & & -1.16 & {$[-2.95,0.63]$} & -1.28 \\
\hline Friendliness & 1.52 & {$[0.65,2.39]$} & $3.42 * * * *$ & 0.39 & {$[-0.89,1.68]$} & 0.60 \\
\hline Openness & 1.54 & {$[0.45,2.63]$} & $2.76 * *$ & 1.97 & {$[-0.03,3.97]$} & 1.93 \\
\hline
\end{tabular}

${ }^{*} p<0.05 .{ }^{* *} p<0.01 . * * * p<0.005 . * * * * p<0.001$.

\section{Reaction Time}

We analyzed RT data with a series of linear mixed models. In light of the previous result and the generally weak correlations between personality and RT, we used a backward selection procedure, removing the lowest scored predictor from the previous model, for all models built on RT data. We first examined the fit of models predicting RT for all first responses (Table 8).

The log-likelihood indicated that model 1, featuring all personality predictors, was the best fit, but AICc suggested that removing Activity added a small improvement in fit. Comparing the two models' predictors directly (Table 9) yields consistent results. In model 2, removing Activity drastically increased the $\chi^{2}$ scores of all predictors, but the two predictors which are significant in model 1 , Confidence and Friendliness, were stronger than all other personality predictors in model 2 . Confidence demonstrated a negative relationship, such that more confident monkeys tended to have lower, i.e., faster, reaction times. Friendliness had an opposite, positive relationship with reaction time; friendlier monkeys were slower to respond.

Table 8

Model Selection Results for Regressions Predicting Log Transformed RTs

\begin{tabular}{|c|c|c|c|c|c|c|c|}
\hline Model Number & Parameters & $\mathrm{df}$ & $\mathrm{AICc}$ & $\mathrm{BIC}$ & LogLik & $\Delta$ LogLik & $\mathrm{RM}$ \\
\hline 0 & (Trial, Intercept) & 9 & 10689.3 & 10751.2 & -5335.6 & & \\
\hline 1 & All & 15 & 10684.4 & 10787.6 & -5327.1 & 8.5 & 0 \\
\hline 2 & Frd,Opn,Con,Anx,Dom & 14 & 10683.4 & 10779.7 & -5327.7 & -0.6 & 1 \\
\hline 3 & Frd,Opn,Con,Dom & 13 & 10684.3 & 10773.7 & -5329.1 & -2.0 & 1 \\
\hline
\end{tabular}

Only the correct first responses were separately analyzed, as well, for these two RT measures may tie into different processes (Prinzmetal et al., 2005). The models' log-likelihoods again suggested that model 1, containing all predictors, was the best fit (Table 10). On the other hand, model 3, containing 
Friendliness, Openness, Confidence, and Dominance, was suggested to be the best fit by AICc. We directly compared these two models and the intermediate model (Table 11).

All three models indicated that Friendliness, Openness, and Confidence were significantly associated with RT on correct first responses. As in our models of all first responses, Friendliness was positively associated with RT, and Confidence negatively associated. Openness demonstrated a negative relationship with $\mathrm{RT}$.

Table 9

Details of Linear Regression Models of Interest, Predicting RTs from Personality

Model $1 \quad$ Model 2

\begin{tabular}{lcccccc}
\hline Predictor & $b$ & $95 \% C I$ & $\chi^{2}$ & $b$ & $95 \% C I$ & $\chi^{2}$ \\
\hline (Intercept) & 3.85 & {$[2.43,5.27]$} & & 3.86 & {$[2.34,5.31]$} & $57.90 * * *$ \\
Trial & -0.011 & {$[-0.013,-0.009]$} & $51.50 * * *$ & -0.011 & {$[-0.013,-0.009]$} & $5.33 *$ \\
Anxiety & -0.60 & {$[-1.08,-0.11]$} & 1.32 & -0.58 & {$[-1.08,-0.09]$} & \\
Activity & 0.17 & {$[-0.15,0.49]$} & 0.25 & & & $23.10 * * *$ \\
Confidence & -0.91 & {$[-1.28,-0.55]$} & $5.71 *$ & -0.91 & {$[-1.29,-0.54]$} & $14.90 * * *$ \\
Dominance & 0.43 & {$[0.11,0.75]$} & 1.66 & 0.52 & {$[0.25,0.79]$} & $44.50 * * *$ \\
Friendliness & 0.56 & {$[0.33,0.79]$} & $5.66 *$ & 0.65 & {$[0.46,0.84]$} & $5.95 *$ \\
Openness & -0.43 & {$[-0.79,-0.07]$} & 1.45 & -0.44 & {$[-0.79,-0.09]$} & 5.05 \\
\hline
\end{tabular}

Note. $p$-values are from Wald's $\chi^{2}$ tests.

$* p<0.05$. ${ }^{* *} p<0.001$.

Table 10

Model Selection Results for Regressions Predicting Log Transformed Rts on Only Correct Trials

\begin{tabular}{|c|c|c|c|c|c|c|c|}
\hline Model Number & Parameters & $\mathrm{df}$ & $\mathrm{AICc}$ & $\mathrm{BIC}$ & LogLik & $\Delta \operatorname{LogLik}$ & $\mathrm{RM}$ \\
\hline 0 & (Trial, Intercept) & 9 & 6217.3 & 6276.2 & -3099.6 & & \\
\hline 1 & All & 15 & 6212.2 & 6310.3 & -3091.0 & 8.6 & 0 \\
\hline 2 & Frd,Opn,Con,Anx,Dom & 14 & 6210.6 & 6302.2 & -3091.3 & -0.3 & 1 \\
\hline 3 & Frd,Opn,Con,Dom & 13 & 6209.2 & 6294.3 & -3091.6 & -0.6 & 1 \\
\hline 4 & Frd,Opn,Con & 12 & 6214.8 & 6293.3 & -3095.4 & -4.4 & 1 \\
\hline
\end{tabular}

\section{Sensitivity Analysis}

To determine if our findings were unique to a six component structure, we extracted our own structures. Because we had only 9 subjects, four methods commonly used to choose how many factors to extract did not yield consistent results. Ruscio and Roche's comparison data, Horn's parallel analysis, Velicer's MAP criterion, and the acceleration factor, as well as two prior studies (Capitanio, 1999; Weiss et al., 2011), suggested anywhere from two to six factors. 
Table 11

Details of Linear Regression Models of Interest, Predicting RTs on Correct Trials

\begin{tabular}{|c|c|c|c|c|c|c|c|c|c|}
\hline & & Model 1 & & & Model 2 & & & Model 3 & \\
\hline Predictor & $b$ & $95 \% C I$ & $\chi^{2}$ & $b$ & $95 \% C I$ & $\chi^{2}$ & $b$ & $95 \% C I$ & $\chi^{2}$ \\
\hline (Intercept) & 4.03 & {$[2.24,5.825]$} & & 4.03 & {$[2.17,5.89]$} & & 3.43 & {$[2.33,4.53]$} & \\
\hline Trial & -0.009 & {$[-0.011,-0.007]$} & $39.60 * * *$ & -0.009 & {$[-0.013,-0.005]$} & $38.90 * * *$ & -0.009 & {$[-0.013,-0.005]$} & $37.60 * * *$ \\
\hline Anxiety & -0.30 & {$[-0.92,0.32]$} & 0.91 & -0.30 & {$[-0.93,0.34]$} & 0.85 & & & \\
\hline Activity & 0.15 & {$[-0.264,0.56]$} & 0.49 & & & & & & \\
\hline Confidence & -0.82 & {$[-1.28,-0.35]$} & $12.00 * * *$ & -0.82 & {$[-1.30,-0.34]$} & $11.30 * * *$ & -0.64 & {$[-0.88,-0.40]$} & $27.50 * * *$ \\
\hline Dominance & 0.29 & {$[-0.12,0.70]$} & 1.94 & 0.37 & {$[0.03,0.71]$} & $4.44 *$ & 0.22 & {$[0.08,0.35]$} & $10.30 * *$ \\
\hline Friendliness & 0.68 & {$[0.39,0.97]$} & $20.80 * * *$ & 0.75 & {$[0.50,0.10]$} & $34.90 * * *$ & 0.76 & {$[0.49,1.02]$} & $32.00 * * *$ \\
\hline Openness & -0.85 & {$[-1.30,-0.40]$} & $13.80 * * *$ & -0.84 & {$[-1.29,-0.39]$} & $13.50 * * *$ & -1.03 & {$[-1.36,-0.70]$} & $37.10 * * *$ \\
\hline
\end{tabular}

Note. $p$-values are from Wald's $\chi^{2}$.

${ }^{*} p<0.05$. ${ }^{* *} p<0.005 . * * * p<0.001$.

Since the interpretation of any single factor structure extracted from these data would be dubious, we used regularized exploratory factor analysis (Jung \& Lee, 2011), a procedure developed for small samples, to separately extract $2,3,4,5$, and 6 factor structures. Salient loadings were defined as $\geq|0.6|$, to minimize cross-loadings. Unit-weighted, varimax rotated matrices were compiled from the salient loadings for each solution. As in prior studies (e.g., Weiss et al., 2011), when more than one factor was salient for an item, the weight was assigned to the factor with the higher loading.

Within every solution, one factor correlated with subjects' averages of our accuracy measures. Which adjectives loaded onto these factors is shown in Table 12. The adjectives 'innovative' and 'inventive', which were each correlated with the averages of our performance measures $(r \mathrm{~s}>|0.84|, p \mathrm{~s}<$ 0.05, after Holm-Bonferroni correction), were salient for all structures. 'Intelligent,' the third adjective to pass Holm-Bonferroni correction, was weighted on only three correlated factors. 'Curious' and 'decisive,' two adjectives correlated with Openness and Friendliness, pre-correction, were salient on three domains, as were 'individualistic,' 'independent,' and 'quitting,' items that were not part of Openness or Friendliness.

Across structures, performance metrics were compared to 20 factors. After Holm-Bonferroni correction, we found that correlations between the accuracy measures and the sixth factor of the six factor structure remained significant. Correlations also maintained significance with the second factor of the two factor structure. Significant correlations were not supported for Rewarded trials, Progress, or Error. These factors were composed largely of the same adjectives (Table 12), some of those explicitly noted in the preceding paragraph. Inclusion of adjectives like 'innovative,' 'inventive,' 'intelligent' and 'curious' represent behaviors associated with openness and intellect. 'Conventional' (negatively loaded), 'individualistic,' 'independent,' and 'decisive' emphasize assertiveness and individuality, monkeys that were extraordinary and whose personalities stood out to our raters. All-together, the traits associated with serial cognitive performance appear to indicate that higher scoring monkeys were more sociable, exploratory, extraordinary, and open. 
Table 12

Common Correlated Items across Five Exploratory Factor Structures

\begin{tabular}{|c|c|c|c|c|c|}
\hline Adjective & 2 & 3 & 4 & 5 & 6 \\
\hline Affectionate & & + & & & \\
\hline Conventional & & & - & - & - \\
\hline Cool & & & - & - & \\
\hline Curious & + & + & + & & \\
\hline Decisive & + & + & & & + \\
\hline Dependent/Follower & & & - & - & \\
\hline Depressed & - & & & & \\
\hline Erratic & & & + & + & \\
\hline Excitable & + & & & & \\
\hline Friendly & & + & & & \\
\hline Helpful & & + & & & \\
\hline Independent & + & & + & + & \\
\hline Individualistic & + & & + & + & \\
\hline Innovative & + & + & + & + & + \\
\hline Inquisitive & + & & + & & \\
\hline Intelligent & + & + & & & + \\
\hline Inventive & + & + & + & + & + \\
\hline Persistent & + & & & & + \\
\hline Playful & + & & + & & \\
\hline Quitting & - & - & & & - \\
\hline Sensitive & & + & & & \\
\hline Sociable & + & + & & & \\
\hline Stingy/Greedy & & & + & + & \\
\hline Sympathetic & + & + & & & \\
\hline Thoughtless & & - & & & \\
\hline Unemotional & - & & & & \\
\hline
\end{tabular}

\section{Discussion}

Rhesus macaques' personalities covary with SimChain task performance: across different measures, Friendliness and Openness were related to performance. These associations extended beyond a priori assumptions about personality structure. Distinct adjectives clustered around factors which consistently correlated with accuracy. 
Openness and Friendliness drive distinct aspects of SimChain performance. Friendliness was consistently related to performance over time: the magnitude of asymptotic performance under the Progress metric, and the linear slope of the transformed Error variable, approaching zero (Figure 2). Openness was related to the rate of learning: the steepness of the Progress curve, and the starting point of the Error curve.

The Error models are not clearly interpretable because we needed to model a transformed Error variable in order to cope with Error's inherent non-linearity. Nevertheless, the contributions of Friendliness and Openness are also distinct in these models. The distinction between the effects of different personality dimensions is lost in our models of Rewarded trials and RTs, and considering that the averages of all accuracy measures are very highly correlated, it may be that a single latent variable drives the relationships between performance and both Openness and Friendliness. This is consistent with the observation that the $g$ factor predicts performance across diverse mental tasks, while being consistently related to personality (Ackerman \& Heggestad, 1997).

Confidence, while not strongly correlated with any performance measure ( $r \mathrm{~s}=0.08$ to 0.38 ), repeatedly appeared as a significant predictor, particularly in models of RT. Researchers of general intelligence recognize that external variables, such as speed-accuracy trade-off strategies and assessment anxiety, can affect assessment (Chamorro-Premuzic \& Furnham, 2014). Confidence appears to be one such variable, being more closely associated with RTs than accuracy; suggesting that it may play a similar role as Extraversion and Neuroticism, associated with speed-accuracy trading-off and test-taking anxiety respectively, in humans. This is consistent with the fact that Confidence captures situational and social fear (Weiss et al., 2011).

Our results compare favorably to those of Morton et al. (2013), who found correlations between Openness and both task participation and response error in capuchin monkeys. Similarly, chimpanzee participation and performance (Herrelko et al., 2012; Hopper et al., 2014), has been tied to the Openness dimension of that species. However, Morton et al. warn against over-extensive comparisons between studies, as neither personality dimensions nor cognitive tasks tend to be directly analogous to one another. Even if personality dimensions have been assigned the same descriptive names post-hoc, they will never represent quite the same capacities. Similarly, while all cognitive tasks will tap into general and more specific domains of intelligence, for researchers to understand the psychological differences underlying individual and species level differences in performance, task implementation must be as consistent as possible.

While animal studies have only begun to explore the associations between personality and cognitive abilities, the literature on humans is more developed, and ought to be used as one reference point for the formulation of hypotheses and interpretation of results. Openness in humans is modestly to moderately correlated with $g$ (Ackerman \& Heggestad, 1997), particularly with typical intellectual engagement and crystalized intelligence. Macaque Friendliness does not have a clear analog among the Big Five; it is mostly constituted by adjectives associated with the human domain of Extraversion and Agreeableness, and perhaps crucially, the item 'intelligent', which positively loads on human Openness (DeYoung, 2014). Monkeys scoring high on Friendliness have been described as "sociable and cooperative" (Weiss et al., 2011, p. 77), and it is likely the cooperative aspect of the domain that makes friendly monkeys strong performers.

In humans, RT has been repeatedly correlated with $g$ (Jensen, 2006). The fact that correct RTs are predicted by Openness and Friendliness is consistent with a general factor among this species. However, the association between Friendliness and RT is positive (i.e., Friendlier monkeys are slower to respond), in contrast to Openness, which has a negative relationship with RT. Friendliness and Openness mirror each other in predicting accuracy. This divergence is curious, but consistent with the hypothesis that RT and accuracy require different mechanisms (Prinzmetal et al., 2005), and suggests that the mechanism underlying the association between RT and $g$ ought to be studied further. RT measures within the human species have proven to be robust, and this study suggests that RT differences could be useful among other primates, but only as a within-species measure. Washburn and Rumbaugh (1997) previously discussed the 
comparative flaws in using RT; to grasp the magnitude and significance of cognitive differences between species, researchers must take care when choosing their measures.

Cognitive and neurological evidence indicates that RT and accuracy rely on different architectures (Landau, Esterman, Robertson, Bentin, \& Prinzmetal, 2007). What evidence we found reinforces this theory; our results imply that RT can be predicted by personality domains that are not related to accuracy. Our findings strengthen the need for comprehensive, unified testing of primate intelligence, particularly in the context of personality, and we reiterate Morton et al.'s (2013) call for caution when studying animal cognition and personality with small samples.

The Primate Cognition Test Battery (Herrmann et al., 2010) is perhaps the best known collection of cognitive tests for primates, but its assessment of physical and spatial cognition is limited to basic, concrete tests; it contains no test of symbolic reasoning, of which SimChain is but one. The SimChain paradigm has been used in several species (Terrace, 1993; Wagner, Hopper, \& Ross, 2015), with immature and adult individuals (Inoue \& Matsuzawa, 2009); the task is repeatable and informative. Distinct cognitive tasks are likely to tap into general or domain specific intelligences to varying degrees, and since it is not known how many factors are best for modeling macaque intelligence, it remains an open question which domains SimChain performance draws on. However, even in models of intelligence with more than a general factor, there tends to be significant overlap between specific domains and $g$ (Danner, Hagemann, Schankin, Hager, \& Funke, 2011). While SimChain is likely representative of $g$, the task is at very least a strong indicator of symbolic reasoning. Additionally, our monkeys had achieved mastery with the SimChain task when tested for this study, so task learning effects would not affect results (Vonk \& Povinelli, 2011); this is beneficial since it removes a confound, but it would also be interesting to investigate associations between personality and task acquisition.

More research is needed to determine how tests of serial cognition relate to other tasks, like numerical addition or object transposition (Herrmann et al., 2010). Once relationships between tasks are established, tests of more advanced cognitive faculties could be incorporated into batteries that assess comparable abilities in primates and adult humans. Regardless of whether general intelligence correlates with one of more primate personality dimensions, individual tests - representative of physical, social, or other cognitive proficiencies - might be tied to different personality dimensions, as is suggested in the human literature (Austin et al., 1997; Chamorro-Premuzic \& Furnham, 2014). Additionally, factor models of primate intelligence have been investigated (Herrmann et al., 2010; Hopkins et al., 2014), and the results have been favorable.

Complex cognitive tasks, like Raven's Progressive Matrices, are extensively used in human intelligence testing because of their strong associations with general intelligence and specific abilities (Austin et al., 1997). Raven's Matrices is also a difficult task, which is a major reason why it is an effective test (Raven, 2000). Our study demonstrates that nonhuman primates are capable of completing complex cognitive tasks that have meaningful associations with personality and intelligence, and other, difficult tasks need not be ruled out as being too challenging for primates.

Our study is not without limitations. SimChain tests serial cognition, and consequently only assesses a portion of a monkey's cognitive repertoire. For instance, while SimChain allows us to capture characteristics about accuracy, it is not as well-suited for studying RTs - we could only model the latency between stimuli onset and the first response. Our sample of monkeys also contained only males, and while a representative sample ought to of course include females, evidence from multiple tasks showed no sex differences in any performance metrics among a group of six male and seven female long-tailed macaques (Schmitt et al., 2012). However, Hopper et al. (2014) found differing contributions from personality to male and female chimpanzees' problem solving success, so we ought not to rule out the possibility that performance in female macaques may have a different relationship with personality.

A comprehensive study using large samples would be the best way to tackle task consistency, sex differences, and other sources of variability. Different primate species, all of whom have been rigorously trained and tested in a diverse range of cognitive tasks, ought to be rated for personality, which would allow us to address questions concerning the evolution of general and specific types of intelligence, and the common origins of intelligence and personality. Even a broad study such as this would likely suffer 
from a drawback that our work suffers from as well: these results rely on captive animals, and captive animals may not be representative of the wider population.

Nevertheless, captive animals are useful models. Rhesus macaques are the gold standard for primate research in neuroscience, genetics, and medicine and our results have implication for these fields. Subjective well-being and personality are heritable and phenotypically and genetically correlated in nonhuman primates (e.g., Adams, King, \& Weiss, 2012). Moreover, Friendliness, which is correlated with subjective well-being in macaques (Weiss et al., 2011), is associated with serial intelligence. Subsequent research is needed to determine if the six macaque domains and subjective well-being are heritable, but in humans and chimpanzees, both well-being and personality are heritable, and genetically correlated (Weiss, Bates, \& Luciano, 2008; Weiss, King, \& Enns, 2002); intelligence too is heritable in both ape species (Davies et al., 2011; Hopkins et al., 2014). The existing monkey literature supports the heritability of personality (Brent et al., 2014; Williamson et al., 2003), though as of yet, no substantive evidence supports the heritability of subjective well-being and intelligence in rhesus macaques. More research needs to investigate these questions, for if individual psychological differences are heritable in macaques, artificial breeding and the research coming out of macaque colonies might be improved by selecting for friendly, intelligent, and mentally healthy phenotypes.

Intelligence and personality are the two pillars of differential psychology. Intelligence has for some time been a major subject of study for evolutionary biologists, and personality has recently gained traction among behavioral ecologists and comparative psychologists (Griffin et al., 2015; Weiss \& Altschul, in press). Deeper investigations into primate cognition and personality will enrich both comparative and differential psychology.

\section{Acknowledgements}

This research was supported by National Institute of Mental Health (NIMH) grant R01 MH051153. The authors would like to thank Greg Jensen and all other members of the Columbia University Primate Cognition Lab for providing the personality ratings in this study.

\section{References}

Ackerman, P. L., \& Heggestad, E. D. (1997). Intelligence, personality, and interests: Evidence for overlapping traits. Psychological Bulletin, 121, 219. doi: 10.1037/0033-2909.121.2.219

Adams, M. J., King, J. E., \& Weiss, A. (2012). The majority of genetic variation in orangutan personality and subjective well-being is nonadditive. Behavior Genetics, 42, 675-686. doi: 10.1007/s10519-012-9537-y

Austin, E. J., Deary, I. J., \& Gibson, G. J. (1997). Relationships between ability and personality: Three hypotheses tested. Intelligence, 25, 49-70. doi: 10.1016/S0160-2896(97)90007-6

Bates, D., Maechler, M., Bolker, B., \& Walker, S. (2015). lme4: Linear mixed-effects models using Eigen and S4 (Version 1.1-9) [Software]. Available from https://CRAN.R-project.org/package=lme4

Bouchard, T. J., Jr., \& Loehlin, J. C. (2001). Genes, evolution, and personality. Behavior Genetics, 31, $243-273$. doi: $10.1023 / \mathrm{A}: 1012294324713$

Brent, L. J., Semple, S., MacLarnon, A., Ruiz-Lambides, A., Gonzalez-Martinez, J., \& Platt, M. L. (2014). Personality traits in rhesus macaques (Macaca mulatta) are heritable but do not predict reproductive output. International Journal of Primatology, 35, 188-209. doi: 10.1007/s10764-013-9724-6

Capitanio, J. P. (1999). Personality dimensions in adult male rhesus macaques: Prediction of behaviors across time and situation. American Journal of Primatology, 47, 299-320. doi: 10.1002/(SICI)10982345(1999)47:4<299::AID-AJP3>3.0.CO;2-P

Chamorro-Premuzic, T., \& Furnham, A. (2014). Personality and intellectual competence. New York, NY: Psychology Press.

Chen, S., Swartz, K. B., \& Terrace, H. S. (1997). Knowledge of the ordinal position of list items in rhesus monkeys. Psychological Science, 8, 80-86. doi: 10.1111/j.1467-9280.1997.tb00687.x

Coleman, K., Tully, L. A., \& McMillan, J. L. (2005). Temperament correlates with training success in adult rhesus macaques. American Journal of Primatology, 65, 63-71. doi: 10.1002/ajp.20097 
Crawford, M. P. (1938). A behavior rating scale for young chimpanzees. Journal of Comparative Psychology, 26, 79-91. doi: 10.1037/h0054503

D’Amato, M. R., \& Colombo, M. (1990). The symbolic distance effect in monkeys (Cebus apella). Animal Learning \& Behavior, 18, 133-140. doi: 10.3758/BF03205250

Danner, D., Hagemann, D., Schankin, A., Hager, M., \& Funke, J. (2011). Beyond IQ: A latent state-trait analysis of general intelligence, dynamic decision making, and implicit learning. Intelligence, 39, 323-334. doi: 10.1016/j.intell.2011.06.004

Davies, G., Tenesa, A., Payton, A., Yang, J., Harris, S. E., Liewald, D., ...Deary, I. J. (2011). Genome-wide association studies establish that human intelligence is highly heritable and polygenic. Molecular psychiatry, 16, 996-1005. doi: 10.1038/mp.2011.85

Deaner, R. O., Van Schaik, C. P., \& Johnson, V. (2006). Do some taxa have better domain-general cognition than others? A meta-analysis of nonhuman primate studies. Evolutionary Psychology, 5, 235-240. doi: $10.1177 / 147470490600400114$

Deary, I. J. (2001). Intelligence: A very short introduction. Oxford, UK: Oxford University Press. doi:10.1093/actrade/9780192893215.001.0001

Deary, I. J., Spinath, F. M., \& Bates, T. C. (2006). Genetics of intelligence. European Journal of Human Genetics, 14, 690-700. doi: 10.1038/sj.ejhg.5201588

DeYoung, C. G. (2014). Openness/Intellect: A dimension of personality reflecting cognitive exploration. APA Handbook of Personality and Social Psychology: Personality Processes and Individual Differences, 4, 369-399. doi: 10.1037/14343-017

Ebbinghaus, H. (1913/2014). Memory: A contribution to experimental psychology. Annals of Neurosciences, 20. doi: 10.5214/ans.0972.7531.200408

Fagot, J., Gullstrand, J., Kemp, C., Defilles, C., \& Mekaouche, M. (2014). Effects of freely accessible computerized test systems on the spontaneous behaviors and stress level of Guinea baboons (Papio papio). American Journal of Primatology, 76, 56-64. doi: 10.1002/ajp.22193

Fox, J., \& Weisberg, S. (2011). An R companion to applied regression ( $2^{\text {nd }}$ edition) [Software]. Available from http://socserv.socsci.mcmaster.ca/jfox/Books/Companion

Goodall, J. (1990). Through a window: 30 years of observing the Gombe chimpanzees. London, England: Weidenfeld \& Nicolson.

Griffin, A. S., Guillette, L. M., \& Healy, S. D. (2015). Cognition and personality: An analysis of an emerging field. Trends in Ecology \& Evolution, 30, 207-214. doi: 10.1016/j.tree.2015.01.012

Guillette, L. M., Hahn, A. H., Hoeschele, M., Przyslupski, A., \& Sturdy, C. B. (2015). Individual differences in learning speed, performance accuracy and exploratory behaviour in black-capped chickadees. Animal Cognition, 18, 165-178. doi: 10.1007/s10071-014-0787-3

Hebb, D. O. (1946). Emotion in man and animal: An analysis of the intuitive process of recognition. Psychological Review, 53, 88-106. doi: 10.1037/h0063033

Herrelko, E. S., Vick, S., \& Buchanan-Smith, H. M. (2012). Cognitive research in zoo-housed chimpanzees: Influence of personality and impact on welfare. American Journal of Primatology, 74, 828-840. doi: 10.1002/ajp.22036

Herrmann, E., Call, J., Hernández-Lloreda, M. V., Hare, B., \& Tomasello, M. (2007). Humans have evolved specialized skills of social cognition: The cultural intelligence hypothesis. Science, 317, 1360-1366. doi: $10.1126 /$ science. 1146282

Herrmann, E., Hernández-Lloreda, M. V., Call, J., Hare, B., \& Tomasello, M. (2010). The structure and individual differences in the cognitive abilities of children and chimpanzees. Psychological Science 21, 102-110. doi: $10.1177 / 0956797609356511$

Hopkins, W. D., Russell, J. L., \& Schaeffer, J. (2014). Chimpanzee intelligence is heritable. Current Biology, 24, 1649-1652. doi: 10.1016/j.cub.2014.05.076

Hopper, L. M., Price, S. A., Freeman, H. D., Lambeth, S. P., Schapiro, S. J., \& Kendal, R. L. (2014). Influence of personality, age, sex, and estrous state on chimpanzee problem-solving success. Animal Cognition, 17, 835-847. doi: 10.1007/s10071-013-0715-y

Inoue, S., \& Matsuzawa, T. (2009). Acquisition and memory of sequence order in young and adult chimpanzees (Pan troglodytes). Animal cognition,12, 59-69. doi: 10.1007/s10071-009-0274-4

Itani, J. (1957). Personality of Japanese monkeys. Iden, 11, 29-33.

Jensen, A. R. (2006). Clocking the mind: Mental chronometry and individual differences. Oxford, UK: Elsevier. 
Jensen, G., Altschul, D., Danly, E., \& Terrace, H. (2013). Transfer of a serial representation between two distinct tasks by rhesus macaques. PLoS one, 8, e70285. doi: 10.1371/journal.pone.0070285

Jung, S., \& Lee, S. (2011). Exploratory factor analysis for small samples. Behavior Research Methods, 43, 701-709. doi: 10.3758/s13428-011-0077-9

King, J. E., \& Figueredo, A. J. (1997). The five-factor model plus dominance in chimpanzee personality. Journal of Research in Personality, 31, 257-271. doi: 10.1006/jrpe.1997.2179

Konečná, M., Lhota, S., Weiss, A., Urbánek, T., Adamová, T., \& Pluháček, J. (2008). Personality in free-ranging Hanuman langur (Semnopithecus entellus) males: Subjective ratings and recorded behavior. Journal of Comparative Psychology, 122, 379-389. doi: 10.1037/a0012625

Landau, A. N., Esterman, M., Robertson, L. C., Bentin, S., \& Prinzmetal, W. (2007). Different effects of voluntary and involuntary attention on EEG activity in the gamma band. The Journal of Neuroscience, 27, 1198611990. doi: 10.1523/JNEUROSCI.3092-07.2007

Matzel, L. D., Han, Y. R., Grossman, H., Karnik, M. S., Patel, D., Scott, N., ...Gandhi, C. C. (2003). Individual differences in the expression of a "general" learning ability in mice. The Journal of Neuroscience, 23, 6423-6433. Retrieved from: http://www.ncbi.nlm.nih.gov/pubmed/12878682.

McCrae, R. R., \& John, O. P. (1992). An introduction to the five-factor model and its applications. Journal of Personality, 60, 172-215. doi: 10.1111/j.1467-6494.1992.tb00970.x

McGonigle, B., \& Chalmers, M. (2006). Ordering and executive functioning as a window on the evolution and development of cognitive systems. International Journal of Comparative Psychology, 19, $241-267$. Retrieved from: http://escholarship.org/uc/item/21m346kb.

Morton, F. B., Lee, P. C., \& Buchanan-Smith, H. M. (2013). Taking personality selection bias seriously in animal cognition research: A case study in capuchin monkeys (Sapajus apella). Animal Cognition, 16, 677-684. doi: 10.1007/s10071-013-0603-5

Pavlov, I. P. (1908/1941). Lectures on conditioned reflexes: Conditioned reflexes and psychiatry (Vol. 2) (W. H. Gantt, Trans.). London: Lawrence and Wishart Limited.

Paxton, R., Basile, B. M., Adachi, I., Suzuki, W. A., Wilson, M. E., \& Hampton, R. R. (2010). Rhesus monkeys (Macaca mulatta) rapidly learn to select dominant individuals in videos of artificial social interactions between unfamiliar conspecifics. Journal of Comparative Psychology, 124, 395-401. doi: $10.1037 / \mathrm{a} 0019751$

Pederson, A. K., King, J. E., \& Landau, V. I. (2005). Chimpanzee (Pan troglodytes) personality predicts behavior. Journal of Research in Personality, 39, 534-549. doi: 10.1016/j.jrp.2004.07.002

Penke, L., Denissen, J. J., \& Miller, G. F. (2007). Evolution, genes, and inter-disciplinary personality research. European Journal of Personality, 21, 639-665. doi: 10.1002/per.657

Pinheiro, J., Bates, D., DebRoy, S., Sarkar, D., \& R Core Team (2015). nlme: Linear and nonlinear mixed effects models (Version 3.1-120) [Software]. Available from: http://CRAN.R-project.org/package=nlme

Prinzmetal, W., McCool, C., \& Park, S. (2005). Attention: Reaction time and accuracy reveal different mechanisms. Journal of Experimental Psychology, 134, 73. doi: 10.1037/0096-3445.134.1.73

R Core Team (2013). R: A language and environment for statistical computing [Software]. Vienna, Austria: R Foundation for Statistical Computing. Available from: http://www.R-project.org/.

Raven, J. (2000). The Raven's progressive matrices: Change and stability over culture and time. Cognitive Psychology, 41, 1-48. doi:10.1006/cogp.1999.0735

Réale, D., Reader, S. M., Sol, D., McDougall, P. T., \& Dingemanse, N. J. (2007). Integrating animal temperament within ecology and evolution. Biological Reviews, 82, 291-318. doi: 10.1111/j.1469-185X.2007.00010.x

Revelle, W. (2015). Psych: Procedures for personality and psychological research (Version 1.5.8) [Software]. Available from: http://CRAN.R-project.org/package=psych

Reader, S. M., Hager, Y., \& Laland, K. N. (2011). The evolution of primate general and cultural intelligence. Philosophical Transactions of the Royal Society B: Biological Sciences, 366, 1017-1027. doi: 10.1098/rstb.2010.0342

Scarf, D., Danly, E., Morgan, G., Colombo, M., \& Terrace, H. S. (2011). Sequential planning in rhesus monkeys (Macaca mulatta). Animal cognition, 14, 317-324. doi: 10.1007/s10071-010-0365-2

Schmitt, V., Pankau, B., \& Fischer, J. (2012). Old World monkeys compare to apes in the primate cognition test battery. PLoS one, 7, e32024. doi: 10.1371/journal.pone.0032024

Shrout, P. E., \& Fleiss, J. L. (1979). Intraclass correlations: Uses in assessing rater reliability. Psychological Bulletin, 86, 420-28. doi: 10.1037/0033-2909.86.2.420 
Terrace, H. S. (1993). The phylogeny and ontogeny of serial memory: List learning by pigeons and monkeys. Psychological Science, 4, 162-169. doi: 10.1111/j.1467-9280.1993.tb00481.x

Vonk, J., \& Povinelli, D. (2011). Individual differences in long-term cognitive testing in a group of captive chimpanzees. International Journal of Comparative Psychology, 24, 137-167.

Vrieze, S. I. (2012). Model selection and psychological theory: A discussion of the differences between the Akaike information criterion (AIC) and the Bayesian information criterion (BIC). Psychological Methods, 17, 228. doi: $10.1037 / \mathrm{a} 0027127$

Wagner, K., Hopper, L., \& Ross, S. (2015). Asymmetries in the production of self-directed behavior by chimpanzees and gorillas during a computerized cognitive test. Animal Cognition, 1-8. doi: 10.1007/s10071-015-0937-2

Washburn, D. A., \& Rumbaugh, D. M. (1997). Faster is smarter, so why are we slower? A comparative perspective on intelligence and processing speed. American Psychologist, 52, 1147-1148. doi:10.1037/0003066X.52.10.1147

Weiss, A., Adams, M. J., Widdig, A., \& Gerald, M. S. (2011). Rhesus macaques (Macaca mulatta) as living fossils of hominoid personality and subjective well-being. Journal of Comparative Psychology, 125, 72-83. doi: $10.1037 / \mathrm{a} 0021187$

Weiss, A., \& Altschul, D. M. (in press). Methods and applications of animal personality research. APA Handbook of Comparative Psychology.

Weiss, A., Bates, T. C., \& Luciano, M. (2008). Happiness is a personal(ity) thing: the genetics of personality and well-being in a representative sample. Psychological Science, 19, 205-210. doi: 10.1111/j.14679280.2008.02068.x

Weiss, A., Inoue-Murayama, M., Hong, K.-W., Inoue, E., Udono, T., Ochiai, T., ...King, J. E. (2009). Assessing chimpanzee personality and subjective well-being in Japan. American Journal of Primatology, 71, 283292. doi: 10.1002/ajp.20649

Weiss, A., King, J. E., \& Enns, R. M. (2002). Subjective well-being is heritable and genetically correlated with dominance in chimpanzees (Pan troglodytes). Journal of Personality and Social Psychology, 83, 1141. doi: 10.1037/0022-3514.83.5.1141

Williamson, D. E., Coleman, K., Bacanu, S. A., Devlin, B. J., Rogers, J., Ryan, N. D., \& Cameron, J. L. (2003). Heritability of fearful-anxious endophenotypes in infant rhesus macaques: A preliminary report. Biological Psychiatry, 53, 284-291. doi: 10.1016/S0006-3223(02)01601-3

Yeo, I. K., \& Johnson, R. A. (2000). A new family of power transformations to improve normality or symmetry. Biometrika, 87, 954-959. doi: 10.1093/biomet/87.4.954

Yerkes, R. M. (1939). The life history and personality of the chimpanzee. The American Naturalist, 73, 97-112. doi: $10.1086 / 280820$ 